\title{
Point Protection of Primary Beams of Steel Grillages Against Lateral Torsional Buckling
}

\author{
Rafał Piotrowski ${ }^{* *}$, Monika Siedlecka ${ }^{1}$ \\ 1 Kielce University of Technology, Faculty of Civil Engineering and Architecture, \\ al. Tysiąclecia Państwa Polskiego 7, 25-314 Kielce, Poland \\ * Corresponding author's e-mail: rafalp@tu.kielce.pl
}

\begin{abstract}
The paper deals with the analysis of lateral torsional buckling (LTB) of steel beams that are components of grillages. Such structures are constructed from primary substring beams and secondary coupling beams. One of the functions performed by secondary beams in grillages is point protection of primary beams against overall stability failure. On the basis of the LTB analysis of primary beams, the condition specified in the code PN-EN 1993-1-1 (EC-3) was verified. The condition concerns the maximum spacing of point lateral bracings that stabilise the compression flanges of beams. Critical moments of LTB for those beams were determined employing two methods. The first method involved the use of equivalent (simplified) loading diagram and the analytic formula developed by the authors. The other method employed the LTBeamN and Abaqus (FEM) software, in which the whole beam was modelled together with bracings. The beam loading diagrams most frequently used in engineering practice were taken into account. Coefficients of LTB were determined in accordance with EC-3. A good congruence of results was obtained, which confirmed the correctness of the equivalent (simplified) beam loading diagram. The condition specified in the code PN-EN 1993-1-1 (EC-3) was also confirmed.
\end{abstract}

Keywords: critical moment of lateral torsional buckling of beams, steel grillages, point protection

\section{INTRODUCTION}

The paper concerns lateral torsional buckling (LTB) analysis of beams in steel grillages (Fig. 1), in which both primary (substrings) beams and also coupling beams are prone to overall stability failure.

The task of the grillage secondary beams (Fig. 1) is to ensure a coaction in the transfer of loads between primary beams. Additionally, the secondary beams provide point protection to primary beams against the overall stability failure, i.e. LTB. In order to account for point support of substring beams of the grillage, it is necessary to use an adequate solution as regards the connections between the beams. In the study, it was assumed that beam joints were designed in such a way that this issue was addressed.

The advantages of point protection of grillage primary beams against LTB were discussed, among others, in studies $[8,12,14]$. Discrete bracings of beams can be divided into those that restrict the lateral displacements of beams in the LTB plane $[12,14]$ and those that restrict the torsion of beams $[8,12,14]$. The effectiveness of the bracing intended to counteract the lateral displacements depends on their location over the height of beam section. The greatest effectiveness is found for the bracing located in the plane of compression (usually top) flange of beams. However, the restraint of the tension (usually bottom) flange produces a small effect on an increase in the lateral torsional buckling resistance of beams. In turn, the advantageous effect of point bracing that restricts the beam torsion is virtually independent of the ordinate of their location. The effectiveness of point protection options of beams against LTB depends on the number of bracings and their location along the beam length. Adequate design of point bracings enables to simultaneously restrict 
lateral displacement and torsion of beams. The stability analysis of braced grillage beams, for different arrangements and bracing degrees, can be conducted for individual beams $[8,14]$ or the whole structure [12].

In this study, the point bracings that prevent the lateral displacement in the LTB plane of compression (top) flanges of primary beams were taken into account. It was assumed that due to the adequate bracing system, secondary beams provided stable support to primary beams.

The LTB (lateral torsional buckling) of primary beams in steel grillages was analysed. The criterion, specified in PN-EN 1993-1-1 (EC-3) [5], concerning the maximum spacing of point lateral bracings that stabilise the top compression flanges of the beams of concern was verified. The critical moments of LTB for primary beams were evaluated using two methods: a) FEM, where the numerical solution was obtained with LTBeamN [6] and Abaqus [1] software tool, in which primary beams were modelled together with point lateral bracings, and b) analytical method, in which an equivalent loading diagram was applied and the author-developed formula was used.

\section{Lateral torsional buckling of steel beams}

Lateral torsional buckling resistance of beam elements that are laterally unbraced is expressed by formula [5]:

$$
M_{b, R d}=\chi_{L T} W_{y} \frac{f_{y}}{\gamma_{M 1}}
$$

where: $\chi_{L T}-$ LTB coefficient;
$W_{y}-$ section resistance index in bending around axis $y-y$, dependent on section class;

$f_{y}-$ yield strength;

$\gamma_{M 1}$ - partial coefficient $\left(\gamma_{M 1}=1.0\right)$.

The value given by the formula (1) depends on the material, the section geometry and LTB coefficient $\chi_{L T}$. In accordance with [5], the LTB coefficient can be evaluated according to a general or special case.

The general case concerns the beams with a constant cross-section, for which LTB coefficient is expressed by formula [5]:

$$
\chi_{L T}=\frac{1}{\Phi_{L T}+\sqrt{\Phi_{L T}^{2}-\bar{\lambda}_{L T}^{2}}} \leq 1.0
$$

where: $\Phi_{L T}=0,5\left[1+\alpha_{L T}\left(\bar{\lambda}_{L T}-0,2\right)+\bar{\lambda}_{L T}^{2}\right]-$ LTB curve parameter; $\alpha_{L T}-$ imperfection factor for LTB; $\bar{\lambda}_{L T}=\sqrt{\frac{W_{y} f_{y}}{M_{c r}}}$-relative slenderness for LTB; $M_{c r}$ - critical moment for elastic LTB.

Special case concerns rolled and welded Ibeams, and allows the estimation of coefficient $\chi_{L T}$ acc. formula [5]:

$$
\chi_{L T}=\frac{1}{\Phi_{L T}+\sqrt{\Phi_{L T}^{2}-\beta \bar{\lambda}_{L T}^{2}}} \leq\left\{\begin{array}{c}
1.0 \\
\frac{1}{\bar{\lambda}_{L T}^{2}}
\end{array}\right.
$$

where: $f=1-0,5\left(1-k_{c}\right)\left[1-2,0\left(\bar{\lambda}_{L T}-0,8\right)^{2}\right] \leq 1.0 \quad-$ LTB curve parameter.

The code [5] recommends to adopt the following parameters: $\bar{\lambda}_{L T, 0}=0.4 \quad$ (maximum

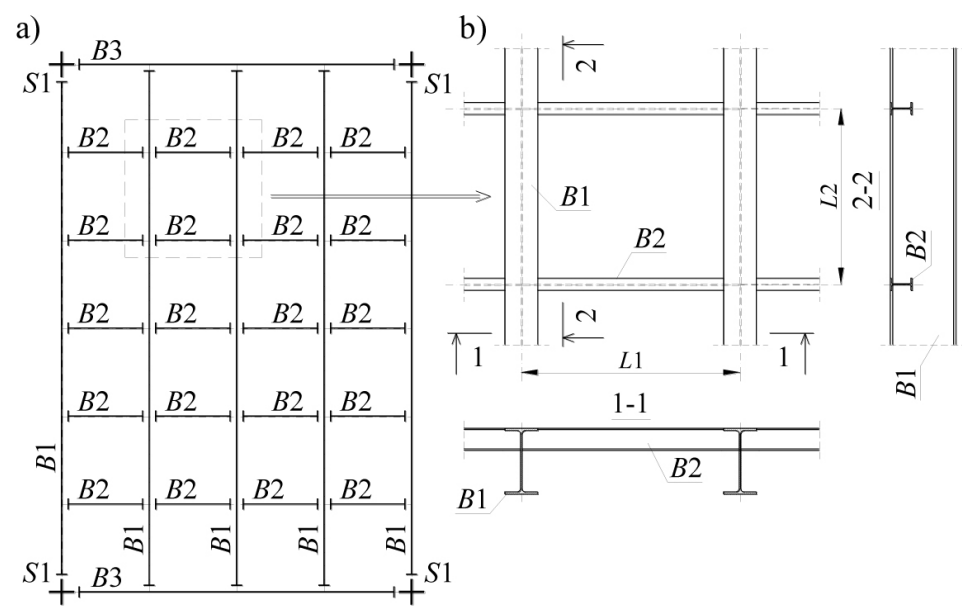

Fig. 1. Exemplary grillage: a) diagram, b) selected grillage fragment. (B1, B3 - primary beams, B2 - secondary beams, S1 - columns) 
value) - maximum slenderness ratio for LTB of rolled sections, $\beta=0.75$ (minimum value) - correction parameter of the LTB curve for rolled sections.

The second case offers a relevant advantage that the distribution of the bending moment between lateral bracings can be taken into account. A modified LTB coefficient [5] can be used to this end:

$$
\chi_{L T, \text { mod }}=\frac{\chi_{L T}}{f} \leq\left\{\begin{array}{c}
1.0 \\
\frac{1}{\bar{\lambda}_{L T}^{2}}
\end{array}\right.
$$

where: $f=1-0,5\left(1-k_{c}\right)\left[1-2,0\left(\bar{\lambda}_{L T}-0,8\right)^{2}\right] \leq 1.0$;

$k_{c}-$ coefficient that accounts for the distribution of moments acc. [5].

In the case of bisymmetric I-beams, often used in engineering practice, while estimating LTB coefficient, the designer can use both formula (2) and formulas (3a) or (3b). On the basis of the subject literature analysis, among others [9], and the author's calculations, it was found that the values of coefficient $\chi_{L T}$ obtained from formula (2) are lower than from formulas (3a) or (3b). Therefore, the application of formulas (3a) or (3b) gives lower reduction in the load capacity of beams, which results in more economic design.

Regardless of which method is employed, in order to determine LTB coefficient $\chi_{L T}$, it is necessary to estimate the critical moment of LTB of beam. The value of the moment depends, among others, on: 1) boundary conditions, 2) the distribution of the bending moment over the beam, and 3 ) the ordinate of the site at which the transverse load is applied along the beam section height. In standard [4], and in many studies on the analysis of stability failure of beams under planar bending, among others, in $[2,7,9,10,11,13]$, the formulas for the critical moment of LTB of fork supported beams loaded in the way most frequently found in the engineering practice were provided.

\section{Point protection of beams against LTB}

In accordance with the code [5], the beams with properly braced compression flange, and also the beams with closed circular or square crosssection are not susceptible to LTB. Continuous or point bracing systems are available for the compression flange of beams. As regards steel grillages, point supports of primary (substrings) beams come in the form of secondary (coupling) beams.

In order to account for point protection of substrings beams against LTB, it is required that the condition of spacing of coupling beams should be satisfied [5]:

$$
\bar{\lambda}_{f}=\frac{k_{c} L_{c}}{i_{f, Z} \lambda_{1}} \leq \bar{\lambda}_{c 0} \frac{M_{c, R d}}{M_{y, E d}}
$$

where: $k_{c}$ - correction coefficient, dependent on moment distribution; ;sep: $L_{c}$ - spacing of lateral bracings that stabilise the compression flange of beams;

$i_{f, z}-$ radius of inertia of the section of the equivalent flange, consisting of the compression flange and $1 / 3$ of the compressed part of the web, with respect to $z-z$ axis of the section;

$\lambda_{1}=93.9 \varepsilon-$ comparative slenderness for compression;

$M_{c, R d}$ - bending resistance of the beam section;

$M_{y, E d}$ - maximum design bending moment between bracings;

$\bar{\lambda}_{c 0}$ - maximum slenderness ratio of the beam compression flange.

In the code [5], a certain discrepancy is found as regards how maximum slenderness ratio $\bar{\lambda}_{c 0}$ of the beam compression flange was specified. This is related to the following:

a) in section 6.3.2.4 (1)B [5] it was stated that:

"Maximum slenderness ratio $\bar{\lambda}_{c 0}$ can be provided in the National Annex. It is recommended to assume that $\bar{\lambda}_{C 0}=\bar{\lambda}_{L T, 0}+0.1$, see 6.3.2.3.", which for $\bar{\lambda}_{L T, 0}=0.4$ (acc. 6.3.2.3) gives $\bar{\lambda}_{c 0}=0.5$;

b) in the National Annex [5] it was stated that: "It is recommended to assume the maximum value $\bar{\lambda}_{L T, 0}=0.4 "$.

Simple transformation of formula (4) produces dependence (5) that makes it possible to determine the maximum spacing of secondary beams, at which acc. [5], primary beams are not in danger of LTB:

$$
L_{c} \leq \frac{i_{f, z} \lambda_{1} \bar{\lambda}_{c 0}}{k_{c}} \frac{M_{c, R d}}{M_{y, E d}}
$$

\section{Critical moment of LTB Mcr}

This study aims to check whether, provided that the spacing of secondary beams in the grillage determined from formula (5) is maintained, primary beams are protected against LTB (i.e. if $\left.\chi_{L T} \geq 1\right)$. The analysis covered simply supported beams (Fig. 2), point-braced at 1/4, 1/2 and 3/4 of 
the span, respectively. Concentrated $\left(P_{z}\right)$ or uniformly distributed $\left(q_{z}\right)$ load was applied to the top flanges of beams $\left(z_{g}=+h / 2\right)$.

The spans of primary beams of grillages were selected based on the spacings of secondary beams, determined with formula (5), i.e. $L=4 L_{c}$ (Fig. 2).

For the analyzed beams, the critical moment of LTB $M_{c r}$ was estimated analytically and determined numerically (FEM).

\section{Mcr estimated analytically (simplified solution)}

For analytical determination of the critical moments of LTB of primary beams, a simplified model was used (Fig. 3). An equivalent loading diagram was employed. The diagram was made for the reduced spans of beams $L_{c}$, specified by the spacing of lateral bracings, in which original loads $\left(P_{z}, q_{z}\right)$ were replaced with concentrated moments applied at the site of beam support. It was assumed that the beams were simply supported.

Critical moments of LTB for equivalent loading diagrams of beams (Fig. 3) were estimated using formula [10]:

$$
M_{c r}=A_{1} \frac{\sqrt{E I_{z}\left(17 L_{c}^{2} G I_{t}+168 E I_{W}\right)}}{L_{c}^{2}}
$$

where: $I_{z}, I_{t}, I_{w}$ - characteristics of beam sections; $L_{c}$ - beam span;

$E, G$ - steel moduli of elasticity; $A_{1}=\frac{19.9}{\sqrt{193+296 \psi+193 \psi^{2}}}-$ coefficient

for the beam loaded with concentrated moments above the supports (coefficient dependent on the loading diagram); $\psi$ - the ratio of support moments $-1 \leq \psi \leq 1$ (Fig. 3).

\section{Mcr determined numerically - FEM}

In order to verify the recommendations in the code (4) [5] and the correctness of the equivalent (simplified) loading diagram used for primary beams (acc. section 4.1), the LTBeamN and Abaqus (FEM) software tools were employed.

LTBeamN (ver. 1.0.3) software [6], based on finite beam elements, is dedicated to the structural engineering applications. It allows the determination of the critical moments of LTB for beams (single-span or continuous, and also cantilevers) with mono- or bi-symmetric I-sections. The tool makes it possible to account, among others, for the following: 1) both classic boundary conditions (fork support or restraint) and also elastic bracings (above the support and over the length of beams as point or continuous bracings), 2) arbitrary number and type of transverse loads, and also loads in the form of axial forces (loads can have an arbitrary ordinate of the application site and arbitrary sense of the force).

Using LTBeam $N$, the analysed beams were modelled for their full span, i.e. $L=4 L_{c}$, taking into account the point lateral bracings in the plane of top flanges of beams. The results of calculations for an exemplary IPE450 beam with span $L=6.88 \mathrm{~m}$ are shown in Figure 4 .

The Abaqus (ver. 6.12) software [1], based on FEM, is used mainly for the research purposes. In this study, it was used for the LTB analysis of exemplary primary beams with point bracing. In modelling, volumetric elements (C3D8) with eight nodes and six degrees of freedom in the node were used. The primary beams were discretized into finite element model with the basic $10 \mathrm{~mm}$ mesh size. The boundary conditions at the supports prevented displacements with respect to the a)

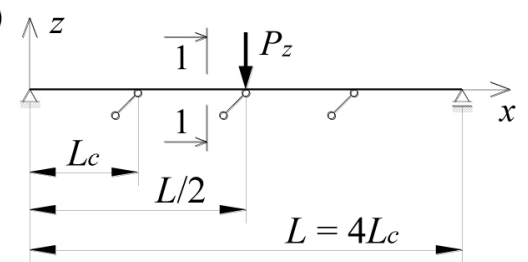

b) $\wedge z$

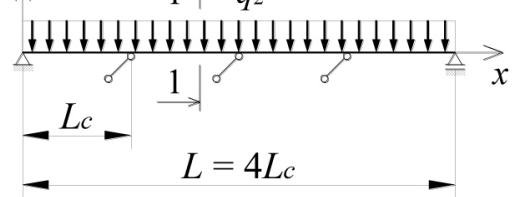

c) 1-1

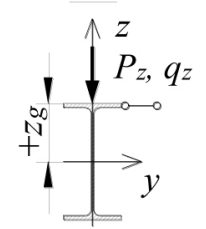

d)

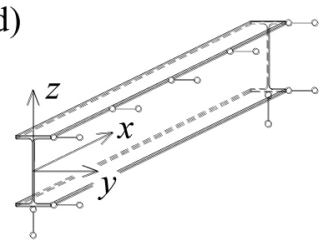

Fig. 2. Diagrams of analysed beams: a) concentrated force load, b) uniformly distributed load, c) cross-section, d) axonometric view. 

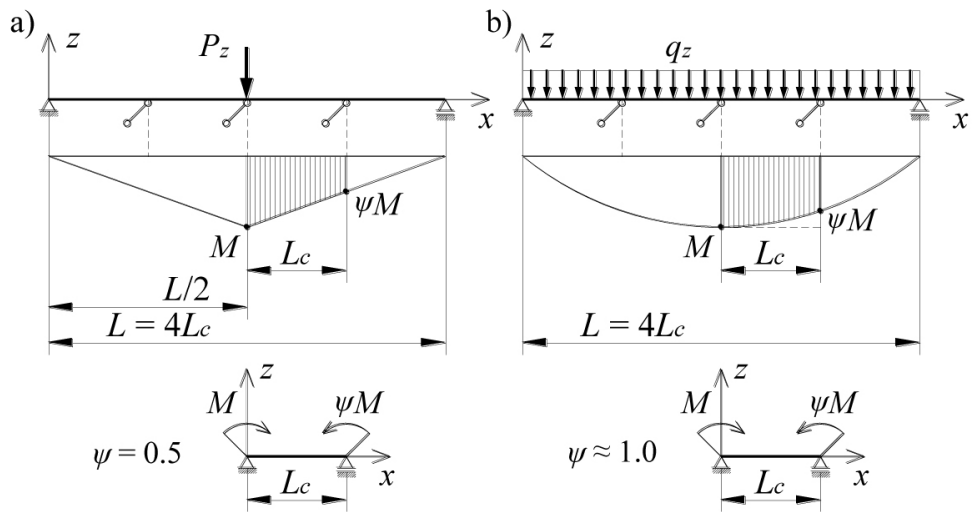

Fig. 3. Equivalent loading diagrams of: a) concentrated force, b) uniformly distributed load.

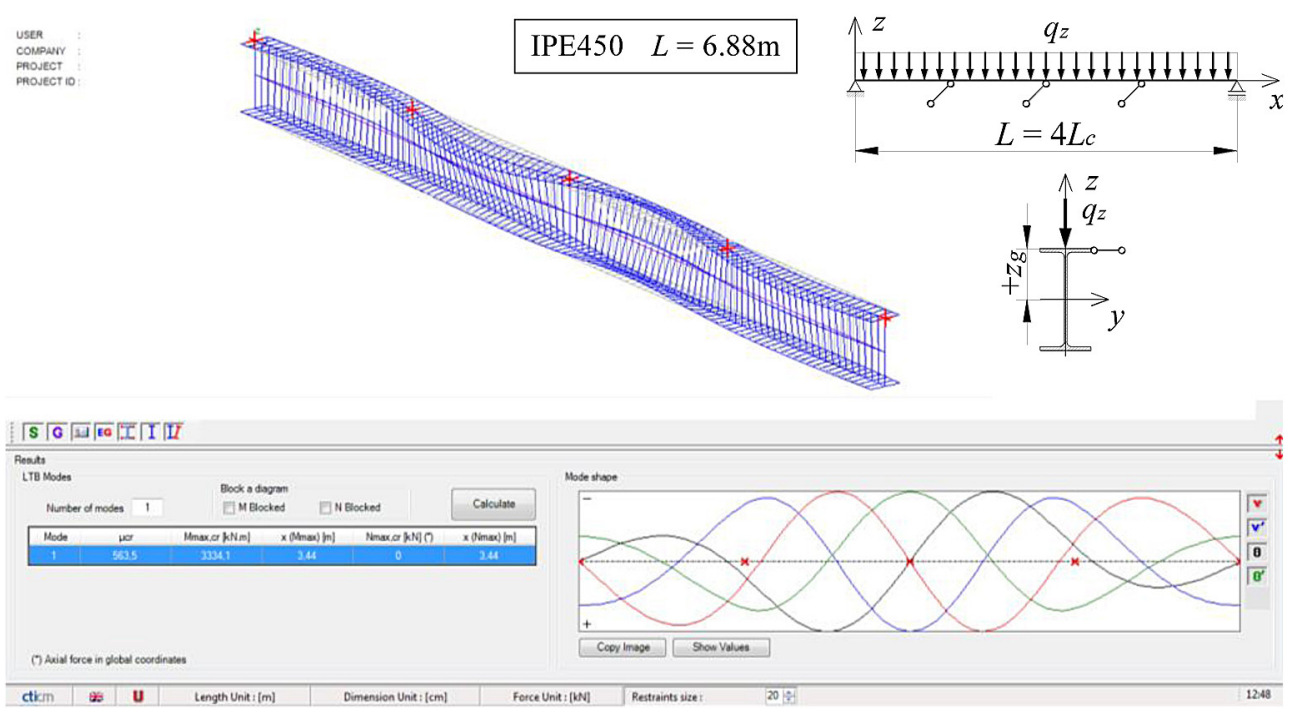

Fig. 4. Exemplary beam modelled with LTBeamN

principal axes of the section inertia, and along the longitudinal axis for one of the supports. The load was applied to the top flange of the beam. The computations were run for the elastic range using the computational step of the buckling procedure.

Figure 5 shows the results of the analysis performed with the Abaqus program for the beam shown in Figure 4.

\section{Examples}

The analysis was conducted for steel beams $(E=210 \mathrm{GPa}, G=81 \mathrm{GPa}, \mathrm{S} 355)$ fabricated from HEB280, IPE450, I400 sections (Table 1) with a span of $L=4 L_{c}$. The exemplary cross-sections of beams (Table 1) were chosen due to the similar value of the section strength index $W_{e l, y^{*}}$ The beams were loaded, in the plane of the top flange, with concentrated force $P_{z}$ or uniformly distributed load $q_{z}$ (Fig. 2). The beam loads $\left(P_{z}, q_{z}\right)$ were applied in the form of unit forces. Primary beams were transversely point-braced by means of secondary beams, which were spaced at distances of $L_{c}$.

When determining the spacing of secondary beams with formula (5), the following assumptions were made (acc. [5]):

a) correction coefficient:

$k_{c}=0.86-$ for the load of a force concentrated at the midspan (Fig. 3a)

$k_{c}=1.0$ - for uniformly distributed load; the coefficient was adopted assuming a constant distribution of bending moment between secondary beams, i.e. for $\psi \approx 1.0$ (Fig. $3 \mathrm{~b}$ )

b) maximum slenderness ratio for the compression beam flange: $\bar{\lambda}_{c 0}=0.5$

As already mentioned (see section 3 ), the code [5] recommends to adopt two values of the slenderness coefficient, i.e. $\bar{\lambda}_{L T, 0}=0.4$ and $\bar{\lambda}_{c 0}=0.5$. The higher value of the coefficient was used in this 


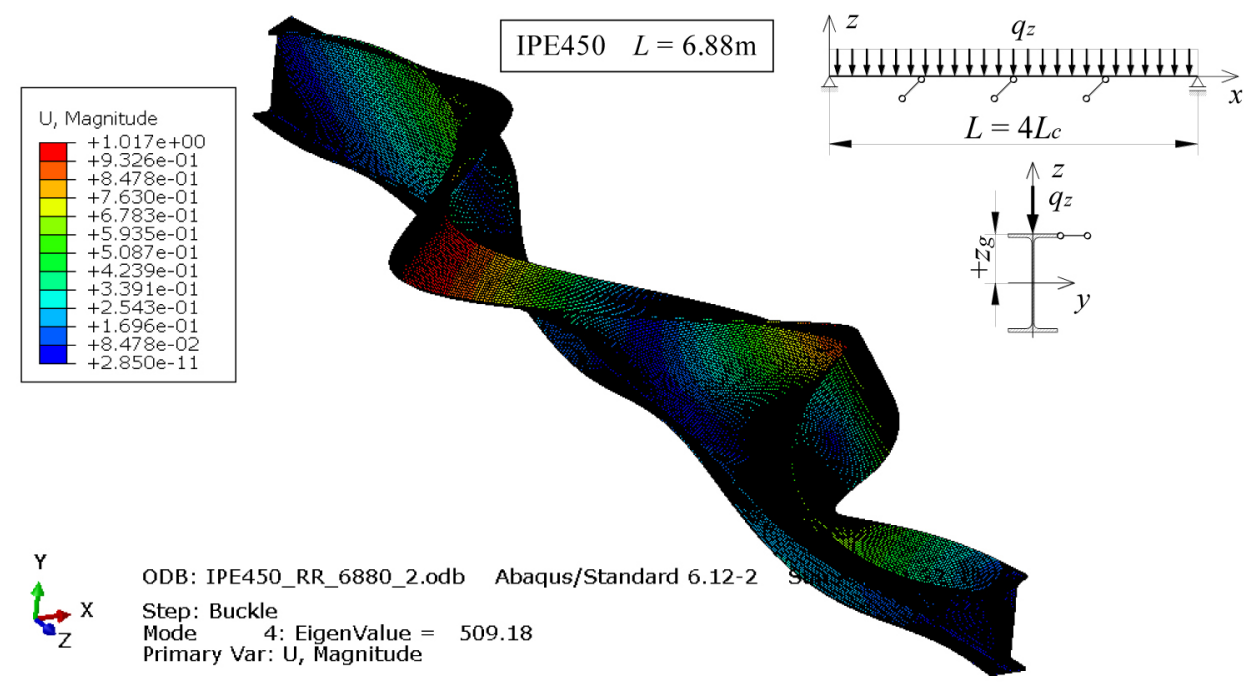

Fig. 5. Exemplary beam modelled with Abaqus

Table 1. Characteristics of sections [3]

\begin{tabular}{|c|c|c|c|c|c|c|}
\hline Item & Profile & $\begin{array}{c}W_{\text {el,y }} \\
{\left[\mathrm{cm}^{3}\right]}\end{array}$ & $\begin{array}{c}I_{z} \\
{\left[\mathrm{~cm}^{4}\right]}\end{array}$ & $\begin{array}{c}I_{w} \\
{\left[\mathrm{~cm}^{6}\right]}\end{array}$ & $\begin{array}{c}I_{t} \\
{\left[\mathrm{~cm}^{4}\right]}\end{array}$ & $\begin{array}{c}i_{f, z} \\
{\left[\mathrm{~cm}^{4}\right]}\end{array}$ \\
\hline 1 & HEB280 & 1380 & 6590 & 1130000 & 144 & 7.48 \\
\hline 2 & IPE450 & 1500 & 1680 & 791000 & 68.9 & 4.52 \\
\hline 3 & 1400 & 1460 & 1160 & 415000 & 183 & 3.65 \\
\hline
\end{tabular}

study, because the spacing of secondary beams $\left(L_{c}\right)$ increases along with the coefficient value. For example, for HEB200 beam, loaded with concentrated force $P_{z}$ in the middle of the span, for $\bar{\lambda}_{c 0}=0.5$ it was obtained $L_{c}=3.324 \mathrm{~m}$, while for $\bar{\lambda}_{L T, 0}=0.4$ the distance was $L_{c}=2.659 \mathrm{~m}$. With respect to the primary beams, this is a more unfavourable variant, because it provides for a larger span of the beams $\left(L=4 L_{c}\right)$.

c) comparative slenderness in compression: $\lambda_{1}=76.435$ (for S355)

d) complete utilisation of resistance of beams: $M_{c, R d}=M_{y, E d}$

Table 2 lists the determined spacings of secondary beams and total spans of primary beams of grillages.
For the spacings of secondary beams shown in Table 2 (column 5) and the spans of primary beams (column 6), the critical moments of LTB of primary beams were estimated from formula (6) [10], and determined with LTBeamN and Abaqus (FEM) (Table 3). When the critical resistances of beams were known, the LTB coefficients were estimated acc. formula (3b) [5].

The coefficients of LTB (Table 3, column 7), calculated on the basis of critical moments determined with LTBeamN and Abaqus (for full spans $L$ of primary beams), confirmed the correctness of formula (4) provided in the code [5]. The critical moments estimated with formula (6) [10] (for reduced spans $L_{c}$ ) produced LTB the coefficients slightly lower than 1 for the uniformly loaded

Table 2. The spans of primary beams of grillages and the spacing of secondary beams

\begin{tabular}{|c|c|c|c|c|c|}
\hline \multirow[t]{2}{*}{ Item } & \multirow[t]{2}{*}{ Loading diagram } & \multirow[t]{2}{*}{ Profile } & \multicolumn{2}{|c|}{$\begin{array}{l}\text { Spacing of secondary beams } \\
L_{c}[\mathrm{~m}]\end{array}$} & \multirow{2}{*}{$\begin{array}{l}\text { Spans of primary beams } \\
\qquad L=4 L_{c}[\mathrm{~m}]\end{array}$} \\
\hline & & & Formula (5) & Assumed & \\
\hline \multirow{3}{*}{1} & \multirow{3}{*}{ 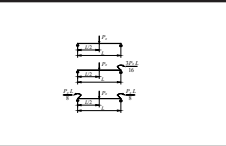 } & HEB280 & 3.324 & 3.320 & 13.280 \\
\hline & & IPE450 & 2.009 & 2.000 & 8.000 \\
\hline & & 1400 & 1.622 & 1.620 & 6.480 \\
\hline \multirow{3}{*}{2} & \multirow{3}{*}{ 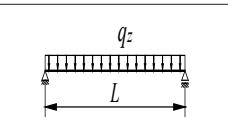 } & HEB280 & 2.859 & 2.850 & 11.400 \\
\hline & & IPE450 & 1.727 & 1.720 & 6.880 \\
\hline & & 1400 & 1.395 & 1.390 & 5.560 \\
\hline
\end{tabular}


beams, and $\chi_{L T, \bmod }=1$ for the beams loaded with a force concentrated at the midspan. It should be noted that LTBeamN and Abaqus accounted for the performance of the whole beam. The edge segments of beam provided elastic stiffening for the segments between the point lateral bracings (middle segments), whereas in formula (6) [10], fork support was assumed. That is reflected in the critical moments of LTB (Table 3, column 6). When determined through LTBeamN, they are greater than those estimated based on formula (6) [10]: for the concentrated force load (Fig. 2a) by approx. $+18 \%$, and for the uniformly distributed load (Fig. 2b) by approx. $+24 \%$. The values of the critical moments of LTB determined in the Abaqus software suite are between those determined with LTBeamN and estimated with formula (6) [10]. A major difference in the critical moments of LTB does not manifest itself in the values of the LTB coefficients (Table 3, column 7).

\section{CONCLUSIONS}

The results listed in Table 3 confirm the correctness of condition (4) specified in the code [5]. For the analysed grillage primary beams, when the spacing of secondary beams does not exceed the values determined through formula (5), the LTB phenomenon can be disregarded when the resistance of beams is determined. However, it should be remembered that secondary beams need to have appropriate rigidity and resistance. In addition, grillage must be provided with proper bracing system. If the loading diagram of beams and the ordinate of point protection of compressed (top) beam flange are changed, or the susceptibility of secondary beams is accounted for, it is necessary to carry out a separate analysis of primary beams adopted in the design.

A good congruence of the results obtained confirms the suitability of the equivalent (simplified) loading diagram (Fig. 3) proposed in the study for the analysed grillage primary beams. However, if it is necessary to take into account the full span of the beam when determining the critical moment of LTB, it is advisable from a technical point of view to use the LTBeamN program.

The analysis of point protection against LTB of the primary beams of the grillage was carried out while taking into account the secondary beam spacing $L_{c}(5)$ specified for slenderness $\bar{\lambda}_{c 0}=0.5$ [5]. For the assumed value of the slenderness coefficient, the correctness of condition (4) [5] was confirmed. In the case coefficient $\bar{\lambda}_{L T, 0}=0.4$ [5] is adopted, a reduction in both the distance $L_{c}$ and the span of the primary beams $\left(L=4 L_{c}\right)$ is obtained. Consequently, to facilitate economical design and structure optimization (among others, a larger span of the primary beams and decrease in the number of secondary beams) slenderness $\bar{\lambda}_{c 0}=0.5$ [5] should be chosen.

Protection against the loss of the flat form of bending of grillage primary beams may

Table 3. Critical moments and LTB coefficients

\begin{tabular}{|c|c|c|c|c|c|c|}
\hline Item & Loading diagram & Profile & Span & \multicolumn{2}{|c|}{$M_{c r}[\mathrm{kNm}]$} & $X_{L T, \text { mod }}(3 \mathrm{~b})[5]$ \\
\hline \multirow{9}{*}{1} & \multirow{4}{*}{ (1) } & \multirow{3}{*}{ HEB280 } & \multirow{2}{*}{$L=13.28 \mathrm{~m}$} & LTBeamN & 3155.70 & 1 \\
\hline & & & & Abaqus & 3020.31 & 1 \\
\hline & & & $L_{c}=3.32 \mathrm{~m}$ & formula (6) [10] & 2674.46 & 1 \\
\hline & & \multirow{3}{*}{ IPE450 } & \multirow{2}{*}{$L=8.00 \mathrm{~m}$} & LTBeamN & 3175.50 & 1 \\
\hline & \multirow{5}{*}{ 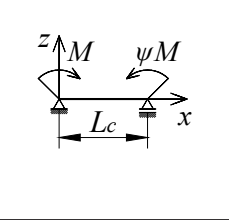 } & & & Abaqus & 2926.90 & 1 \\
\hline & & & $L_{c}=2.00 \mathrm{~m}$ & formula (6) [10] & 2666.75 & 1 \\
\hline & & \multirow{3}{*}{1400} & \multirow{2}{*}{$L=6.48 \mathrm{~m}$} & LTBeamN & 3259.10 & 1 \\
\hline & & & & Abaqus & 2843.97 & 1 \\
\hline & & & $L_{c}=1.62 \mathrm{~m}$ & formula (6) [10] & 2765.49 & 1 \\
\hline \multirow{9}{*}{2} & \multirow{9}{*}{ 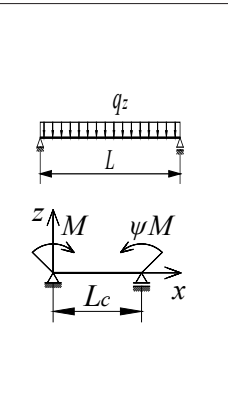 } & \multirow{3}{*}{ HEB280 } & \multirow{2}{*}{$L=11.40 \mathrm{~m}$} & LTBeamN & 3232.20 & 1 \\
\hline & & & & Abaqus & 3027.09 & 0.999 \\
\hline & & & $L_{c}=2.85 \mathrm{~m}$ & formula (6) [10] & 2611.02 & 0.987 \\
\hline & & \multirow{3}{*}{ IPE450 } & \multirow{2}{*}{$L=6.88 \mathrm{~m}$} & LTBeamN & 3334.20 & 1 \\
\hline & & & & Abaqus & 3012.72 & 0.989 \\
\hline & & & $L_{c}=1.72 \mathrm{~m}$ & formula (6) [10] & 2681.21 & 0.974 \\
\hline & & \multirow{3}{*}{1400} & \multirow{2}{*}{$L=5.56 \mathrm{~m}$} & LTBeamN & 3365.50 & 1 \\
\hline & & & & Abaqus & 2913.18 & 0.988 \\
\hline & & & $L_{c}=1.39 \mathrm{~m}$ & formula (6) [10] & 2718.93 & 0.979 \\
\hline
\end{tabular}


significantly increase their critical and limit resistance. Additionally, the need to account for the interaction between the grillage beams in the design is also justified by the economic reasons. Lower steel consumption reduces the costs of project delivery.

In case the condition (4) set in the code [5] is not satisfied, the LTB phenomenon should be accounted for in the analysis of the resistance of primary beams in grillages.

\section{REFERENCES}

1. Abaqus 6.12. PDF Documentation. Dassault Systèmes, 2012.

2. Bijak R. The lateral buckling of simply supported unrestrained bisymmetric I-shape beams. Archives of Civil Engineering, LXI(4), 2015, 127-140.

3. Bogucki W. and Żyburtowicz M. Tables for design of metal structures. Arkady, 2010. (in Polish)

4. [4] Eurocode 3: Design of steel structures. Part 1.1: General rules and rules for buildings; English version ENV 1993-1-1, April 1992 ed.

5. Eurocode 3: Design of steel structures. Part 1.1: General rules and rules for buildings; Polish version PN-EN 1993-1-1, June 2006 ed.

6. Galéa Y. Moment critique de déversement élastique de poutres fléchies. Présentation du logiciel LTBEAM. Revue Construction Métallique. CTICM, 40(2), 2003, 47-76.

7. Kuhlmann U. Stahlbau Kalender: Stabilität, Membrantragwerke. Ernst \& Sohn, 2009.

8. Lee H.E., Nguyen C.T., Moon J.H. and Joo H.S. Lateral-torsional buckling of discretely-braced i-girder. Procedia Engineering, 14, 2011, 264-271.

9. Pałkowski Sz. and Popiołek K. Analysis of lateral torsional buckling of steel beams according to PNEN 1993-1-1. Inżynieria i Budownictwo, 64(6), 2008, 299-302. (in Polish)

10. Piotrowski R. and Szychowski A. Applying the energy method and polynomials to the determination of the critical buckling moments in beams. Konstrukcje betonowe i metalowe, WU UTP w Bydgoszczy, 2015, 249-257. (in Polish)

11. Serna M.A., López A., Puente I. and Yong D.J. Equivalent uniform moment factors for lateraltorsional buckling of steel members. Journal of Constructional Steel Research, 62, 2006, 566-580.

12. Stroetmann R. Lateral torsional and distortional buckling of cross-connected beams. Proc. of 8th International Conference on Advances in Steel Structures, Lisbon, Portugal 2015, 1-12.

13. Trahair N.S., Bradford M.A., Nethercot D.A. and Gardner L. The behavior and design of steel structures to EC3. Taylor \& Francis, 2008.

14. Yura J. Fundamentals of beam bracing. Engineering Journal, 1, 2001, 11-26. 